Check for updates

Cite this: RSC Adv., 2018, 8, 19085

Received 12th March 2018 Accepted 20th April 2018

DOI: $10.1039 / c 8 r a 02153 h$

rsc.li/rsc-advances

\section{Potentiation effect on accelerating diabetic wound healing using 2-N,6-O-sulfated chitosan-doped PLGA scaffold}

\author{
Xiaohui Zhang, (DD) ab Yang Liu, ab Shuang Zhang, ${ }^{\text {ab }}$ Tong Shen, ${ }^{\text {ab }}$ Jing Wang ${ }^{\star a b}$ \\ and Changsheng Liu*abc
}

Accelerating the wound healing of diabetes-impaired cutaneous tissue is still a challenge due to the aberrant cellular behavior, poor angiogenesis, and pathological micro-environment. Activation with growth factors and modulation of the redox micro-environment of the diabetic wound are considered to be effective strategies. Herein, we have described a highly sulfated heparin-like polysaccharide 2-N, 6-O-sulfated chitosan (26SCS)-doped poly(lactic-co-glycolic acid) scaffold (S-PLGA), which can achieve controlled and sustained release of heparin-binding epidermal growth factor (HB-EGF) owing to its affinity for heparin-binding growth factors. Interestingly, the antioxidant effect of 26SCS was confirmed and it was shown to have a strong scavenging activity towards superoxide radicals, a moderate scavenging activity towards hydroxyl radicals and a lower scavenging activity towards hydrogen peroxide. It also exhibited stronger protective effects in a human keratinocyte cell line (Ha-cat) against $\mathrm{H}_{2} \mathrm{O}_{2}$-induced oxidative damage. The Ha-cat cells cultured in the presence of the S-PLGA scaffold were significantly protected against oxidative stress during proliferation. In a full thickness excisional wound model of a diabetic rat, the wound treated with the HB-EGF-loaded S-PLGA scaffold was basically healed after 28 days. Conversely, the wounds in the other diabetic groups were not closed. The migration effect of the keratinocytes was enhanced by the 26SCS-induced sustainable release of HBEGF and the scavenging of ROS which led to rapid re-epithelialization. Furthermore, histopathological evaluation demonstrated the positive effects on wound contraction, epithelial regeneration, and collagen deposition when treated with the HB-EGF loaded S-PLGA scaffold. These findings highlight that 26SCS may serve as a promising coagent for both controlled release of growth factors and alleviation of excessive ROS production, thus leading to increased regeneration of the diabetic wounds.

\section{Introduction}

It is estimated that 285 million people worldwide are affected by diabetes, and $15 \%$ of these patients suffer from chronic ulcers. This figure is expected to rise to 439 million by $2030 .{ }^{1}$ Despite the danger posed by poor healing, very few specific therapies exist, leaving patients at risk of hospitalization, amputation, and further decline in overall health. The underlying mechanism for the formation of diabetic chronic wounds is still unclear. Diabetic chronic wounds are multi-factorial in origin. They are characterised by impaired granulocytic, chemotaxis and macrophage functions, as well as prolonged inflammation

${ }^{a}$ Key Laboratory for Ultrafine Materials of Ministry of Education, East China University of Science and Technology, Shanghai 200237, PR China.E-mail: biomatwj@163.com; liucs@ecust.edu.cn

${ }^{b}$ Engineering Research Center for Biomedical Materials of Ministry of Education, East China University of Science and Technology, Shanghai 200237, PR China

${ }^{c}$ The State Key Laboratory of Bioreactor Engineering, East China University of Science and Technology, Shanghai 200237, PR China and deregulation of the neovascularization phase. ${ }^{2,3}$ There may also be nitric oxide abnormalcy, collagen accumulation, abnormal function of fibroblasts and keratinocytes, as well as accumulation of ECM ingredients and their remodeling by matrix metalloproteinases (MMPs). ${ }^{4-6}$

The treatment of diabetic wounds is mainly limited to standard wound care practices, including surgical debridement, antibiotic therapy, moisture dressing, and pressure off loading, as well as close control of blood glucose levels. ${ }^{7}$ Various approaches, such as the use of growth factors, stem cells, gene therapy, proteins and natural products have been used to promote diabetic chronic wound healing. To accelerate the wound healing process, plenty of dressings are used to protect long-range healing from contamination/infection, to afford an ideal moist environment to stimulate wound healing, to offer controlled and sustained release of the bioactive substances, and to prevent their fast degeneration during the repair process. ${ }^{8}$ Unfortunately, until now, there has been no effective treatment or application method that can adequately ensure chronic wound healing. 
At the wound site, reactive oxygen species (ROS), such as $\mathrm{H}_{2} \mathrm{O}_{2}$, are generated as by-products along with proinflammatory mediators, which play an important role in managing normal wound healing by accelerating hemostasis, inflammation, wound closure, and formation of the extracellular matrix (ECM). ${ }^{9}$ During diabetic wound healing, normal redox signaling is disrupted resulting in raised ROS levels in a variety of tissues, due to the overproduction of ROS by macrophages and neutrophils, and a damaged antioxidant defense ability caused by hyperglycemia. ${ }^{10}$ Excessive ROS production leads to oxidative stress, which can cause impaired dermal fibroblast and keratinocyte function. ${ }^{11,12}$ Some studies have also reported that ROS can stimulate the degradation of hypoxia-inducible factor 1 (HIF-1), which reduces the expression of HIF- $1 \alpha$-dependent genes such as vascular endotheliumderived growth factor, hemoxygenase-1 (HO-1), and endothelial and inducible nitric oxide synthase. ${ }^{13,14}$ The increased production of vascular superoxide $\left(\mathrm{O}^{2-}\right)$ in diabetic patients may inactivate nitric oxide (NO), which is detrimental to wound repair. ${ }^{15}$ Furthermore, ROS can enhance the expression of MMPs. An increased ECM degradation rate, resulting from the high level of MMPs, can impair cell attachment, migration and cell signaling. ${ }^{16}$

Notably, modulation of the redox environment of the diabetic wound has proven to be an effective strategy. A number of mechanisms for targeted ROS-generation have been well defined. For example, the topical application of siRNA targeting in xanthine dehydrogenase $(\mathrm{XDH})$ in $\mathrm{db} / \mathrm{db}$ diabetic mice decreased the activity of xanthine oxidase (XO), and significantly improved the healing rate. ${ }^{17}$ Increasing the antioxidant ability has proven to be a valid strategy. The in vivo transfer of siRNA targeted against Keap1, to restore Nrf2 antioxidant function, was shown to accelerate diabetic tissue regeneration. ${ }^{18}$ Non-specific therapy for decreasing ROS has also been probed recently. Antioxidants such as vitamin C, bilirubin ointment and SkO1 help to maintain a balance by reducing ROS levels at the wound site and promoting wound healing..$^{19-21}$

Chitosan (CS) or chitooligosaccharide (COS), a natural polysaccharide, can combine with superoxide free radicals, acting as a natural antioxidant, due to the abundance of free hydroxyl and amino groups. ${ }^{22}$ It was reported that CS, acting as a protective agent against $\mathrm{H}_{2} \mathrm{O}_{2} / \mathrm{FeSO}_{4}$-induced cell death in the NT2 neural cell line, could show potential for the prevention and treatment of some central nervous system (CNS) diseases. ${ }^{23}$ It was also reported that low molecular weight COS exhibits anti-antioxidant effects in $\beta$-cells by scavenging radicals in vitro and can improve insulin secretion due to high glucose levels. ${ }^{24}$ Of particular interest are some chitosan derivatives, such as sulfated chitosan (SCS), which could enhance antioxidant activity. Ronge Xing et al. reported the disparate antioxidant ability of various sulfated chitosans. When comparing chitosan and sulfated chitosan with the same molecular weight, the scavenging activity of sulfated chitosan towards superoxide and hydroxyl radicals was better than that of chitosan..$^{25}$ Moreover, many sulfated polysaccharides have recently been shown to have ambiguous results in preventing oxidative stress. For example, the sulfated derivatives of Artemisia sphaerocephala polysaccharide were found to have a stronger antioxidant activity when compared to the native polysaccharide in vitro. ${ }^{26}$ The sulfated polysaccharides also exhibited better protective effects in RAW264.7 against $\mathrm{H}_{2} \mathrm{O}_{2}$-induced oxidative damage, when compared to the native polysaccharide. ${ }^{27}$ Most of these studies focused on the antioxidative effects of the polysacchrides. However, until now, the precise antioxidative mechanism of sulfated polysaccharides has remained unclear, especially at the cellular level. ${ }^{28}$ The antioxidant activity of 26SCS in diabetic chronic wounds has also not been reported so far.

HB-EGF is a potent stimulator of keratinocyte proliferation and migration during wound healing. Many studies have demonstrated that the application of EGF would accelerate wound healing by shortening the healing time. ${ }^{29}$ However, it must be applied in a sustained and localized way to be efficient due to its short half-life. In a previous work by our group, we showed that a 26SCS-modified carrier could achieve controlled release of HB-EGF with a steady release rate when compared with neat PLGA nanofibers and it also has a synergistic effect on the migration of keratinocytes, which leads to rapid re-epithelialization..$^{30-33}$ However, the application of antioxidative 26SCS in regenerative medicine has not been reported, especially for the treatment of diabetic chronic wounds.

In view of this, we focused on developing a scaffold that would promote the collective migration of keratinocytes and the enhanced formation of granulation tissue. We hypothesize that 26SCS could promote keratinocyte survival and migration and accelerate diabetic wound healing. The effect of 26SCS, serving as a synergetic coagent, on both the controlled release of HBEGF and the survival of normal human keratinocytes upon oxidative stress was investigated. Moreover, the survival upon oxidative stress of the keratinocytes was assessed in the case of the 26SCS-doped PLGA scaffold. Finally, a wound repair examination was implemented to verify the in vivo promotion potency of the 26SCS-doped scaffold using a full-thickness excisional wound model in STZ-diabetic rats.

\section{Experimental}

\subsection{Fabrication of the 26SCS-doped PLGA scaffold}

2.1.1 Electrospinning of PLGA scaffold. The electrospinning solutions were prepared by dissolving $1.5 \mathrm{~g}$ PLGA in a mixed solution, which contained $7.5 \mathrm{~mL}$ tetrahydrofuran and

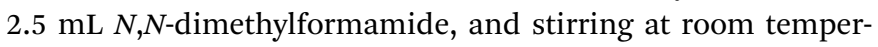
ature until the PLGA completely dissolved. A certain amount of mixed solution was withdrawn with a $10 \mathrm{~mL}$ syringe and embedded into a micro syringe pump a number of times. The solution feed rate was set at $2.5 \mathrm{~mL} \mathrm{~h}^{-1}$. The collector was placed $10 \mathrm{~cm}$ away from the syringe needle tip and the output voltage was set to $18 \mathrm{kV}$. The fibers were dried overnight under vacuum and were then used for further studies.

2.1.2 Aminolysis of PLGA scaffold. The PLGA scaffolds were cut to $1 \mathrm{~cm} \times 1 \mathrm{~cm}$ dimensions, and were then reacted with $0.43 \mathrm{~mol} \mathrm{~L}^{-1}$ 1,6-hexanediamine/isopropanol at $25{ }^{\circ} \mathrm{C}$ for 15 min. The aminolyzed PLGA scaffolds were then thoroughly 
washed in pure water three times and dried in a vacuum oven set at room temperature.

2.1.3 26SCS grafting. A $4 \mathrm{mg} \mathrm{mL} \mathrm{mL}^{-1}$ 26SCS solution was obtained by dissolving 26SCS in sodium citrate buffer solution (pH 4.5). To activate the carboxylic acid groups of 26SCS, $0.15 \mathrm{~mol} \mathrm{~L}^{-1} \mathrm{NHS}$ and $0.30 \mathrm{~mol} \mathrm{~L}^{-1} \mathrm{EDC}$ were added into the buffer solution, and the solution was kept at room temperature for $4 \mathrm{~h}$. Subsequently, the aminolyzed PLGA scaffolds were immersed in the 26SCS buffer solution for $24 \mathrm{~h}$ to obtain the 26SCS-doped PLGA scaffolds (S-PLGA). After the reaction, the scaffolds were washed with buffer and pure water three times to remove the weakly adsorbed $26 \mathrm{SCS}$ and then dried at room temperature.

\subsection{Characterizations of scaffolds}

2.2.1 Morphology observation. The morphology of the electrospun scaffold after 26SCS grafting was characterized using scanning electron microscopy (SEM; S-3400, Hitachi, Tokyo, Japan). The scaffolds were scanned at an accelerating voltage of $10 \mathrm{kV}$ after sputter coating with gold.

2.2.2 Mechanical properties. The scaffolds were cut into similar dimensions $(5 \mathrm{~cm} \times 1 \mathrm{~cm})$ for mechanical testing. ${ }^{34}$ The stress-strain curves of the samples were measured using a tensile tester (HY-0350 Shanghai Hengyi Testing Instruments Co., Ltd) operating at a rate of $1 \mathrm{~mm} \mathrm{~min}^{-1}$. All of the mechanical tests were reported as an average of three measurements.

2.2.3 Contact angle measurements. The hydrophilicity of the PLGA scaffolds and the 26SCS-immobilized PLGA scaffolds was determined using sessile drop water contact angle measurements. The microinjector was spun to ensure each sessile drop was $1 \mu \mathrm{L}$. The data were recorded using a digital camera (Nikon, Tokyo, Japan) after $20 \mathrm{~s}$ of equilibration.

2.2.4 X-ray photoelectron spectroscopy analysis. The surface chemical composition of the aminolyzed PLGA scaffold (N-PLGA) and the 26SCS-immobilized PLGA scaffold (S-PLGA) was investigated using XPS (ESCALAB 250Xi, Thermo Scientific, Waltham, MA, USA). The data were analyzed using XPS Peak Software. The relevant integral peak intensities were calculated to determine the atomic concentration of each element. The $\mathrm{C} 1 \mathrm{~s}$ neutral carbon peak at $285 \mathrm{eV}$ was used as the reference to compensate for surface charging.

2.2.5 Surface charge characterization. The effect of 26SCS modification was investigated by characterizing the distribution of the negative charge on the PLGA scaffolds. Rhodamine 6G (R6G) with positively-charged fluorophores can adsorb on the modified surface through the action of positive and negative charges, and the distribution of the negative charges was measured using a fluorescence microscope. Briefly, the 26SCSimmobilized PLGA scaffold was soaked in phosphate buffer ( $\mathrm{pH}=8.0)$ for $0.5 \mathrm{~h}$. Subsequently, the scaffolds were added to the R6G solution $\left(1.0 \mathrm{mg} \mathrm{mL}^{-1}\right)$ and left to stand for $0.5 \mathrm{~h}$, and then washed with phosphate buffer 3 times. Fluorescence microscopy photographs were taken after drying.

2.2.6 Toluidine blue staining and assay. The amount of 26SCS grafted onto the PLGA scaffold was determined using a toluidine blue assay. Briefly, $0.005 \%(\mathrm{w} / \mathrm{v})$ toluidine blue solution was firstly prepared by dissolving toluidine blue in $0.01 \mathrm{~mol} \mathrm{~L}^{-1}$ hydrochloric acid containing $0.2 \%(\mathrm{w} / \mathrm{v}) \mathrm{NaCl}$. 26SCS solutions $(700 \mu \mathrm{L})$ with known concentrations $(0-50 \mu \mathrm{g}$ $\left.\mathrm{mL}^{-1}\right)$ were reacted with the toluidine blue solution $(300 \mu \mathrm{L})$ for $3 \mathrm{~h}$ at $37^{\circ} \mathrm{C}$. $N$-Hexane $(600 \mu \mathrm{L})$ was used to extract the toluidine blue-26SCS complex into the organic phase by shaking for $1 \mathrm{~min}$. The absorbance of the aqueous solution (containing the unreacted toluidine blue) at $630 \mathrm{~nm}$ was measured using an enzyme-linked immunosorbent, and this was used to construct a calibration curve. To determine the amount of immobilized SCS, the 26SCS-immobilized PLGA scaffold (S-PLGA, $1 \times 1 \mathrm{~cm}^{2}$ ) was immersed in PBS $(600 \mu \mathrm{L})$, and reacted with the toluidine blue solution $(300 \mu \mathrm{L})$ for $3 \mathrm{~h}$ at $37^{\circ} \mathrm{C}$. Subsequently, $n$-hexane was added to ensure the same treatment. After removing the PLGA-SCS-dye complex from solution, the absorbance of the aqueous phase was measured at $630 \mathrm{~nm}$.

2.2.7 HB-EGF release profile. The release behaviour of the scaffolds was measured using a Human HB-EGF ELSIA kit. Briefly, the scaffolds were placed in a 48 -well plate. $10 \mu \mathrm{L}$ of PBS solution containing $1 \mu \mathrm{g}$ HB-EGF was blotted onto each sample and left for $4 \mathrm{~h}$ to be absorbed. Then the HB-EGF loaded scaffolds were lyophilized and placed into $1 \mathrm{~mL}$ PBS in a vertical mouth bottle $(5 \mathrm{~mL})$ at $37{ }^{\circ} \mathrm{C}$ under continuous agitation. At specified time points, $1 \mathrm{~mL}$ release buffer was collected and stored in a freezer, and an equal volume of fresh PBS was added to the bottle. All of the collected samples were thawed and the release behavior was determined using a Human HB-EGF ELISIA kit. All of the samples were tested in triplicate.

\subsection{In vitro antioxidant activity}

2.3.1 Superoxide radical scavenging assay. The superoxide scavenging ability of 26SCS was assessed using the NBT reduction method. ${ }^{35}$ The reaction mixture containing $100 \mu \mathrm{L}$ sample solution $\left(0.05-0.5 \mathrm{mg} \mathrm{mL}^{-1}\right), 300 \mu \mathrm{L}$ PMS $(60 \mu \mathrm{M}), 300$ $\mu \mathrm{L}$ NADH $(468 \mu \mathrm{M})$ and $300 \mu \mathrm{L}$ NBT $(150 \mu \mathrm{M})$, dissolved in phosphate buffer $(0.1 \mathrm{M}, \mathrm{pH}=7.4)$, was incubated at room temperature for $5 \mathrm{~min}$ and the absorbance was measured at $560 \mathrm{~nm}$ against a blank. For the control, the sample was replaced by PBS. VC and TP were used as standards. The capability of scavenging was determined using the following equation: scavenging ability $(\%)=\left[1-\left(A_{\text {sample }}-A_{\text {blank }}\right) / A_{\text {control }}\right]$ $\times 100$.

2.3.2 Hydroxyl radical scavenging assay. The reaction mixture containing $100 \mu \mathrm{L}$ sample solution $\left(1-10 \mathrm{mg} \mathrm{mL}^{-1}\right), 500$ $\mu \mathrm{L}$ deoxyribose $(3.75 \mathrm{mM}), 100 \mu \mathrm{L} \mathrm{H}_{2} \mathrm{O}_{2}(1 \mathrm{mM}), 100 \mu \mathrm{L} \mathrm{FeCl}_{3}$ (100 mM), $100 \mu \mathrm{L}$ EDTA (100 mM) and $100 \mu \mathrm{L}$ ascorbic acid (20 $\mathrm{mM})$, dissolved in phosphate buffer $(20 \mathrm{mM}, \mathrm{pH} 7.4)$, was incubated for $60 \mathrm{~min}$ at $37^{\circ} \mathrm{C} .{ }^{36}$ To terminate the reaction, $1 \mathrm{~mL}$ TBA $(1 \%, w / v)$ and $1 \mathrm{~mL}$ TCA $(2 \%, \mathrm{w} / \mathrm{v})$ were added and then the tubes were heated at $80{ }^{\circ} \mathrm{C}$ for $15 \mathrm{~min}$. When the contents were cooled, $2.0 \mathrm{~mL}$ of 1-butanol was added, and the 1-butanol phase was clarified for the absorbance measurement at $530 \mathrm{~nm}$ against a 1-butanol blank. ${ }^{37}$ The percentage scavenging effect was calculated from the decrease in absorbance against the control (without sample). VC was used as the standard. 
2.3.3 Assay of $\mathrm{H}_{2} \mathrm{O}_{2}$ scavenging. The ability of the samples to remove $\mathrm{H}_{2} \mathrm{O}_{2}$ was determined spectrophotometrically. ${ }^{38}$ The samples were dissolved in $0.1 \mathrm{M} \mathrm{pH} 7.4$ phosphate buffered saline (PBS). The reaction mixture, containing $100 \mu \mathrm{L}$ sample solution (0.05-0.5 mg mL ${ }^{-1}$ ) and $0.6 \mathrm{~mL}$ of $\mathrm{H}_{2} \mathrm{O}_{2}$ solution (40 $\mathrm{mM}$ ), was incubated for $10 \mathrm{~min}$. The absorbance of $\mathrm{H}_{2} \mathrm{O}_{2}$ at $230 \mathrm{~nm}$ was measured using a spectrophotometer. For each concentration, a separate blank sample was used for background subtraction. The $\mathrm{H}_{2} \mathrm{O}_{2}$ scavenging activity was calculated as follows: scavenging rate $(\%)=\left[1-\left(A_{\text {sample }}-A_{\text {blank }}\right) /\right.$ $\left.A_{\text {control }}\right] \times 100$, where $A_{\text {control }}$ is the absorbance of the control group in the hydroxyl radical generation system, $A_{\text {sample }}$ is the absorbance of the test group and $A_{\text {blank }}$ is the absorbance of the samples only.

\subsection{In vitro cell culture studies}

2.4.1 Cell culture. A human keratinocyte cell line (Ha-cat, purchased from American Type Culture Collection) was inoculated in Dulbecco's modified Eagle's medium (DMEM) supplemented with $10 \%$ fetal bovine serum (FBS), and $100 \mathrm{mg} \mathrm{mL}^{-1}$ streptomycin (cell culture reagents were obtained from SigmaAldrich, Saint Louis, MO, USA).

2.4.2 $\mathrm{H}_{2} \mathrm{O}_{2}$ treatment of Ha-cat cells with soluble SCS. The Ha-cat cells were seeded in a 96-well plate $\left(1 \times 10^{4}\right.$ cells per well $)$ for the cell integrity assay, and they were serum starved overnight. The Ha-cat cells were then pre-treated with soluble SCS (50-100 $\mu \mathrm{M})$ for $2 \mathrm{~h}$. The cells were then exposed to DMEM supplemented with $500 \mu \mathrm{M} \mathrm{H}_{2} \mathrm{O}_{2}$ in the presence or absence of SCS, and then the cells were placed into Dulbecco's modified Eagle's medium (DMEM) supplemented with 10\% fetal bovine

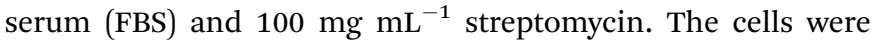
investigated using the MTT test after incubation for $24 \mathrm{~h}$. The absorption values were measured at $570 \mathrm{~nm}$.

2.4.3 $\mathrm{H}_{2} \mathrm{O}_{2}$ treatment of Ha-cat cells on the scaffold. The Ha-cat cells were plated $\left(1 \times 10^{4}\right.$ cells per well $)$ in a 96 -well plate and cultured in the presence of the scaffold for $6 \mathrm{~h}$. The cells were exposed to $\mathrm{H}_{2} \mathrm{O}_{2}$ at a concentration of $100 \mu \mathrm{M}$ for $2 \mathrm{~h}$. Then the cells were placed into Dulbecco's modified Eagle's medium (DMEM) supplemented with 10\% fetal bovine serum (FBS) and $100 \mathrm{mg} \mathrm{mL} \mathrm{mL}^{-1}$ streptomycin. The CCK-8 assay was used to evaluate the proliferative potential of the cells after incubation for $24 \mathrm{~h}$. The absorption values were measured at $570 \mathrm{~nm}$.

\subsection{In vivo assessment using diabetic rat models}

2.5.1 The streptozotocin-induced diabetic rat models. The animal experiments were approved by the Research Ethics Committee at the Shanghai Sixth People's Hospital affiliated to Shanghai Jiao Tong University. All of the animal experiments were conducted in accordance with the guidelines of the Research Ethics Committee of the Sixth People's Hospital, Shanghai Jiao Tong University, and the guide for the Care and Use of Laboratory Animals. Adult male SD rats (Silaike Inc. Shanghai, China, $n=63$ ), with an average body weight of 200$250 \mathrm{~g}$, were rendered diabetic by using a single intraperitoneal injection of $70 \mathrm{mg} \mathrm{kg}^{-1}$ streptozotocin dissolved in $0.1 \mathrm{M}$ citrate buffer $(\mathrm{pH}=4.5)$. The blood glucose levels were measured using
Table 1 Six experimental groups

\begin{tabular}{llll}
\hline Group & Rat & Scaffold & HB-EGF \\
\hline Non-DM blank group & Normal & - & - \\
DM blank group & Diabetic & - & - \\
DM P+0 group & Diabetic & PLGA & - \\
DM S+0 group & Diabetic & S-PLGA & - \\
DM P+4 group & Diabetic & PLGA & $4 \mu \mathrm{g}$ \\
DM S+4 group & Diabetic & S-PLGA & $4 \mu \mathrm{g}$ \\
\hline
\end{tabular}

an electronic device (Glucometer, Accu-Check, Germany) at days 2, 3 and 7. The animals that showed a blood glucose concentration $>16.7 \mathrm{mM}$ were considered to be diabetic. ${ }^{39}$ Two weeks later, the rats were randomly divided into six groups (Table 1), $n=10 \mathrm{rat} / 20$ wounds per group. The dorsal surface of the
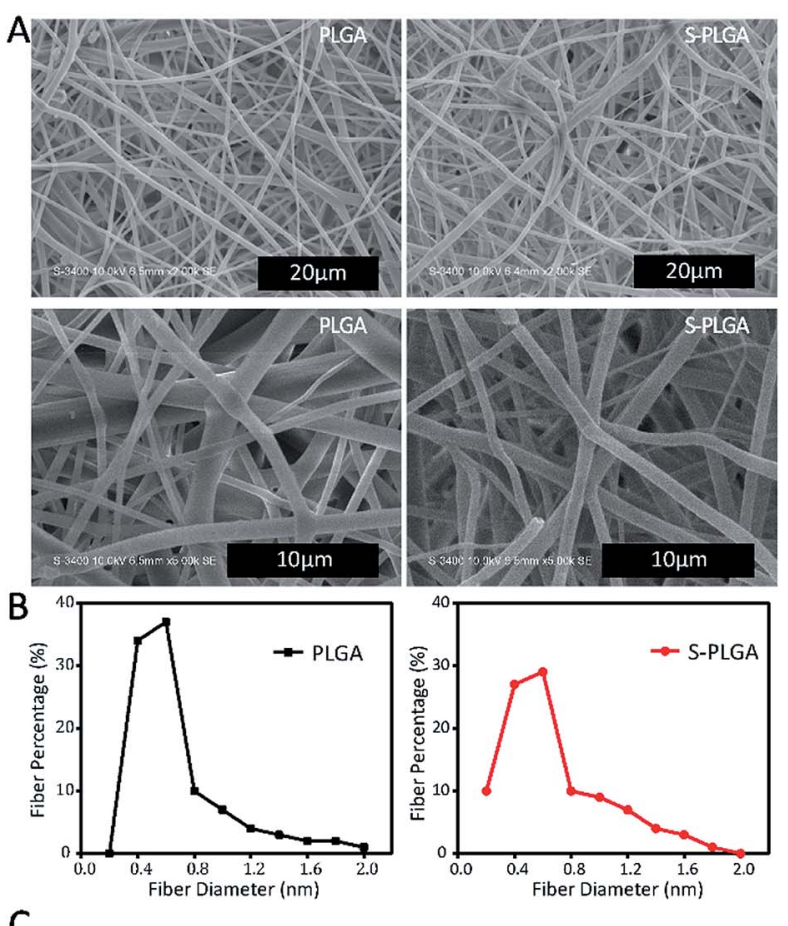

C
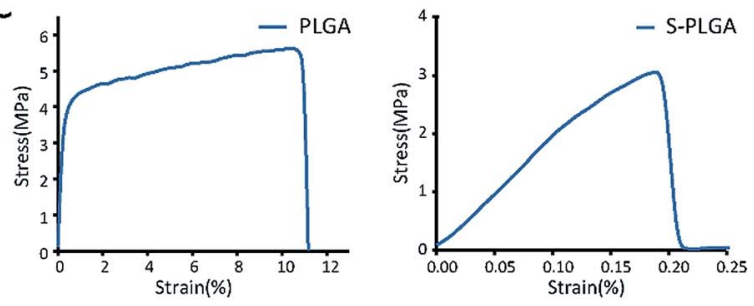

D
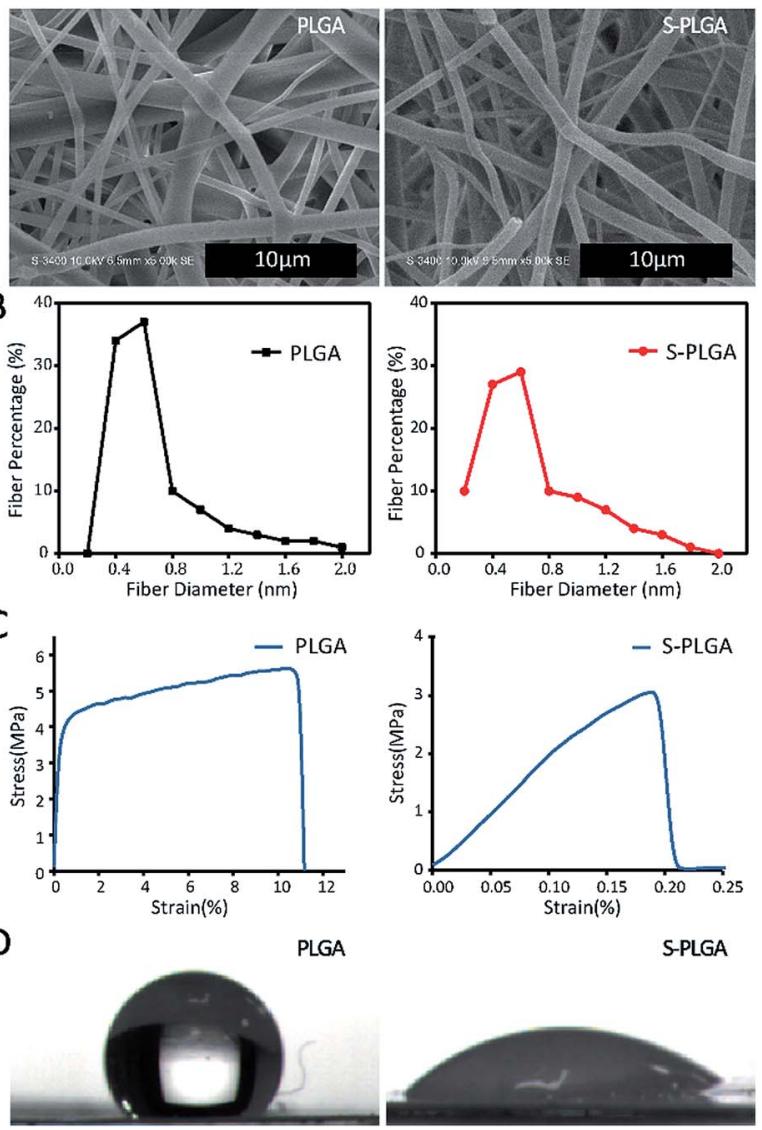

g. 1 (A) SEM micrographs of the PLGA and S-PLGA scaffolds. (B) The fiber diameter of the PLGA and S-PLGA scaffold. (C) The tensile strength of the PLGA and S-PLGA scaffold. (D) The hydrophilicity of the PLGA and S-PLGA scaffold. 
animal was shaved with an electric clipper and cleaned with $75 \%$ alcohol solution. Two circular excisional, full-thickness cutaneous wounds, with diameters of $18 \mathrm{~mm}$ were created on the back of each rat. The scaffold, with a diameter of $18 \mathrm{~mm}$, was implanted and the pledget was sutured around the wound site using 3-0 synthetic absorbable sutures (PGA suture). The pledget was removed after 7 days.

2.5.2 Serial wound analysis. The wound area was calculated after treatment for 7, 14 and 28 days. The skin wounds of the six groups were photographed, and the area of the unhealed wound was determined based on the image using Image pro plus software. The percentage of the wound closure was calculated according to: $\%$ wound closure $=\left(A_{\max }-A_{\mathrm{d}}\right) / A_{\max } \times 100 \%$

where $A_{\max }=$ area of the original wound and $A_{\mathrm{d}}=$ area of the actual wound.

2.5.3 DHE staining analysis. Dihydroethidium (DHE) staining was used to evaluate the intracellular ROS. Briefly, regenerated wound tissue samples, after 7 days post-surgery, were fixed in $4 \%$ paraformaldehyde and sections were made with thicknesses of $10 \mu \mathrm{m}$ using cryotome. The sections were stained with dihydroethidium (DHE) $(10 \mu \mathrm{M})$ solution for $30 \mathrm{~min}$. DHE or hydroethidium is a compound which penetrates into the cells and interacts with $\mathrm{O}^{2-}$ and thereby forms a by-product known as oxyethidium. Upon interaction with nucleic acids, this product emits a red color that can be qualitatively detected using a confocal microscope.

2.5.4 Immunofluorescent analysis. On day 7, the frozen sections were dual stained with rabbit polyclonal antiki-67 (1:100, Abcam) and mouse monoclonal anti-integrin b4 (1 : 100, Abcam), followed by goat anti-rabbit IgG Alexa Fluor 594 ( $1: 200$, Abcam) and goat anti-mouse IgG FITC secondary antibodies (1:200, Abcam), and counterstained with DAPI.

2.5.5 Histological analysis. At days 7, 14 and 28 after the operation, the wound tissue sample sections were treated with $\mathrm{HE}$ and Masson's trichrome staining to observe the wound repair process. The dyed sections were photographed using an inverted microscope. The histological analysis for all of the samples was performed on more than 4 wounds per group at each time point. The images presented are representative of all of the replicates.

2.5.6 Statistical analysis. All of the quantitative data are expressed as the mean \pm SD and were analyzed with Origin 9.0 (OriginLab Corp, USA). Statistical differences were detected using one-way analysis of variance (ANOVA). Statistical significance was accepted at greater than the 95\% confidence level $(p<0.05)$.

\section{Results}

\subsection{Characterization of the scaffold}

The PLGA scaffold showed a well-defined fiber structure with an average fiber diameter of $686 \mathrm{~nm}$ (Fig. 1). After two steps of ammonification and sulfonation, the S-PLGA scaffold showed edge dissolution, and the S-PLGA scaffold showed conglutination and ruptured. The average fiber diameter of the S-PLGA scaffold was $678 \mathrm{~nm}$. The tensile strength of the PLGA scaffold and the S-PLGA scaffold was 5.6 and $3.1 \mathrm{MPa}$, respectively. Typical skin tensile strength ranges from 2 to $16 \mathrm{MPa} .^{40}$ Before and after modification, the tensile strength of the scaffolds was like normal skin. The water contact angle on the S-PLGA scaffold $\left(46.50^{\circ}\right)$ was smaller than that on the PLGA scaffold $\left(128.99^{\circ}\right)$. The hydrophilicity of the scaffold after modification was enhanced.

XPS analysis revealed the successful immobilization of 26SCS (Fig. 2A). In the spectra, the N1s peak was fitted and deconvoluted to help to identify the contribution of the $\mathrm{N}$ element in different chemical environments. The peaks at 401.8 and $399.6 \mathrm{eV}$ are assigned to $-\mathrm{NHSO}_{3}{ }^{-}$, protonated amines (C$\left.\mathrm{NH}_{3}{ }^{+} / \mathrm{C}-\mathrm{N}^{+}\right)$and amides $(\mathrm{N}-\mathrm{C}-\mathrm{O})$, respectively. The N-PLGA scaffold showed a peak at $399.6 \mathrm{eV}$, which is caused by the aminolysis of the PLGA molecular chain, which could produce the amide (N-C-O). ${ }^{49}$ After 26SCS grafting, the scaffold showed
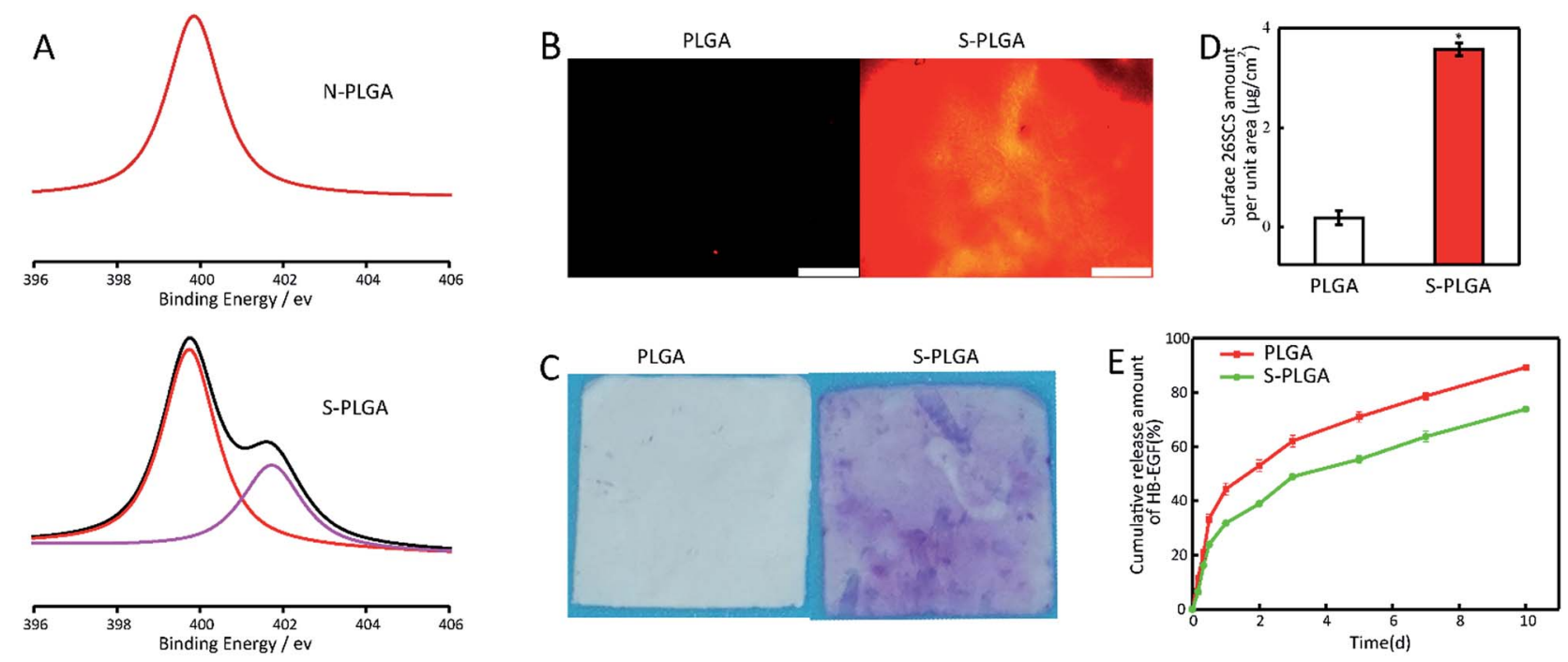

Fig. 2 (A) XPS analysis. The N1s high resolution spectra of the PLGA and S-PLGA scaffold. (B) Characterization of the negative charge on the surface of the PLGA and S-PLGA dressing, scale bar $=500 \mathrm{~nm}$. (C) Appearance of the PLGA and S-PLGA scaffold. (D) The 26SCS-grafting amount on the S-PLGA scaffold, ${ }^{*} p<0.05$. (E) The release kinetics of HB-EGF from the PLGA and S-PLGA scaffolds. 
A

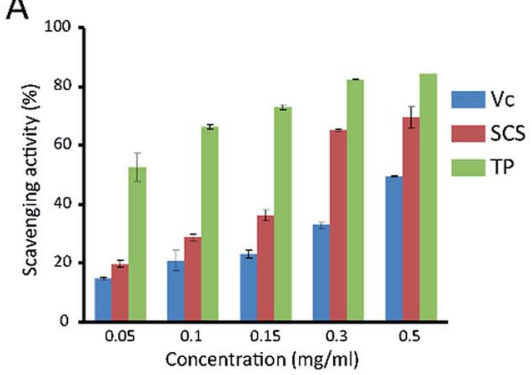

B

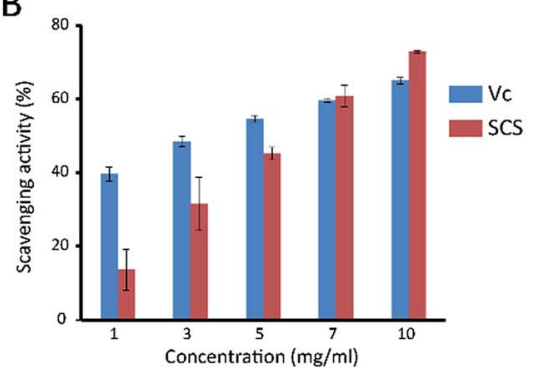

C

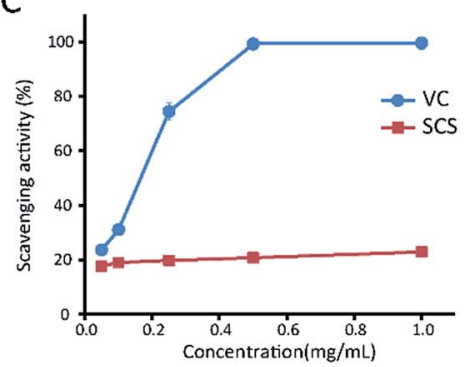

Fig. 3 (A) The scavenging activity towards the superoxide radical, (B) the hydroxyl radical scavenging activity and (C) the $\mathrm{H}_{2} \mathrm{O}_{2}$ scavenging activity.

a new peak at 401.8 , which was caused by the presence of ${ }^{-} \mathrm{NHSO}_{3}{ }^{-}$in $26 \mathrm{SCS} .{ }^{41}$ The nitrogen content of the peak at $399.6 \mathrm{eV}$ increased from $0.63 \%$ to $1.04 \%$, which is caused by the interaction of $-\mathrm{SO}_{3}{ }^{-}$with $-\mathrm{NH}_{3}{ }^{+}$. Further characterization of the negative charge on the surface helped to characterize the surface modification of 26SCS directly. When compared with PLGA, the surface modified with 26SCS shows an obvious distribution of fluorescent charge (Fig. 2B). The presence of 26SCS molecules after covalent immobilization was quantitatively determined using the toluidine blue assay. The PLGA scaffold exhibited a very pale purple color due to the trace physical adsorption of toluidine blue, whereas the 26SCSgrafted PLGA scaffold showed a strong purple color (Fig. 2C). The results of the surface charge and toluidine blue staining analysis show that 26SCS was distributed evenly on the surface of the scaffold. The amount of 26SCS grafted onto the PLGA scaffold was measured as $3.66 \mu \mathrm{g} \mathrm{cm}^{-2}$ (Fig. 2D).

\subsection{Release kinetics of HB-EGF}

The release of HB-EGF was almost linear and was sustained throughout the 10 day experiment. At day 10, a cumulative release of $73.8 \%$ was observed for the S-PLGA scaffold, compared with $89.3 \%$ for the PLGA scaffold, indicating that the sustained release effect of HB-EGF is enhanced after modification (Fig. 2E).

\subsection{In vitro antioxidant activity}

The scavenging of the superoxide radical (19.7-69.6\%) at all of the concentrations $\left(0.05-0.5 \mathrm{mg} \mathrm{mL} \mathrm{m}^{-1}\right)$ of $26 \mathrm{SCS}$ exhibited values between those of $\mathrm{Vc}$ and $\mathrm{TP}$ and this was concentration
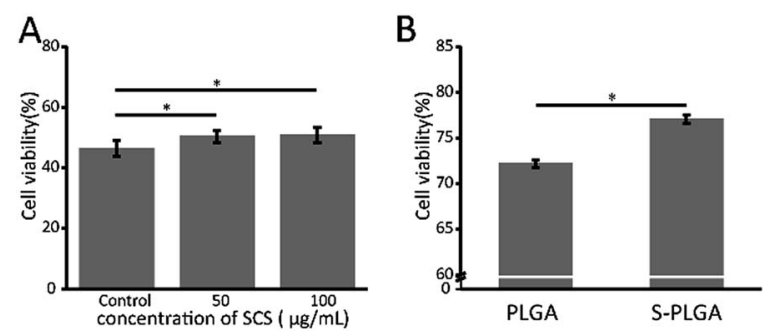

Fig. 4 (A) $\mathrm{H}_{2} \mathrm{O}_{2}$ treatment regimen for the $\mathrm{Ha}$-cat cells in the presence or absence of $26 \mathrm{SCS}$. (B) $\mathrm{H}_{2} \mathrm{O}_{2}$ treatment regimen for the $\mathrm{Ha}$-cat cells in the presence of the PLGA scaffold and S-PLGA scaffold, ${ }^{*} p<0.05$. dependent (Fig. 3A). The scavenging effect of 26SCS towards the hydroxyl radical at different concentrations $\left(1-10 \mathrm{mg} \mathrm{mL}^{-1}\right)$ was between 13.7 and $72.9 \%$. More significant inhibition was observed at high concentrations $\left(7-10 \mathrm{mg} \mathrm{mL}^{-1}\right)$ of 26SCS when compared to Vc (Fig. 3B). However, the hydroxyl radical scavenging activity of Vc was stronger than that of 26SCS at low concentrations (1-5 mg mL${ }^{-1}$ ). In the range of $0.05-1 \mathrm{mg} \mathrm{mL}^{-1}$, the scavenging rate of $26 \mathrm{SCS}$ towards $\mathrm{H}_{2} \mathrm{O}_{2}$ ranged from $17.7 \%$ to $22.9 \%$. For Vc, the rate was $99.7 \%$ at $1 \mathrm{mg} \mathrm{mL} \mathrm{m}^{-1}$, which is higher than that of 26SCS. There were no significant changes in the range of $0.05-1 \mathrm{mg} \mathrm{mL}^{-1}$ (Fig. 3C).

\subsection{Assessment of cell viability in vitro}

The protective effect of 26SCS on the viability of $\mathrm{H}_{2} \mathrm{O}_{2}$-induced Ha-cat cells was identified using the MTT assay. As shown in Fig. $4 \mathrm{~A}$, the viability of the Ha-cat cells in the control group

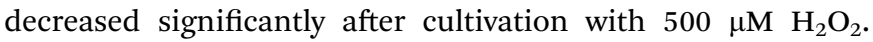
However, the relative viability of the Ha-cat cells pre-protected with 26SCS for $2 \mathrm{~h}$ was significantly increased. We then investigated the effect of the S-PLGA scaffold on Ha-cat cell survival following treatment with $100 \mu \mathrm{M} \mathrm{H}_{2} \mathrm{O}_{2}$. The cck8 assay showed an increased percentage of viable Ha-cat cells in the presence of the S-PLGA scaffold (Fig. 4B).

\subsection{Wound closure measurement}

The effect of HB-EGF loading on wound closure was determined by measuring the area around a wound over 28 days. As displayed in Fig. 5B, at day 7, the HB-EGF-loaded S-PLGA scaffold (DM S+4) group had the vast advantage of quick wound healing when compared with the other DM groups and this was maintained throughout the trial. A dramatic decrease in the wound area was observed in the case of the DM S+4 group with $71 \%$ healing occurring in 7 days. During the same time interval, the wound closure of the other DM groups was no more than $70 \%$. At day 14 , the wounds in the DM S+4 group had healed by about $90 \%$. This still holds the advantage for speeding up wound healing when compared with the other DM groups and it is like the non-DM blank group. Furthermore, epithelialization appeared to have been nearly completed by day 28 . However, the wounds were not completely closed in the other DM groups. In addition, the DM $\mathrm{S}+0$ group showed significantly improved wound closure when compared with the DM blank group and the DM $\mathrm{P}+0$ group. 
A

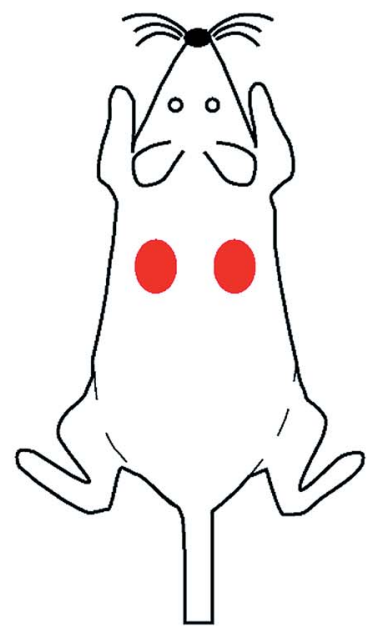

C non-DM blank
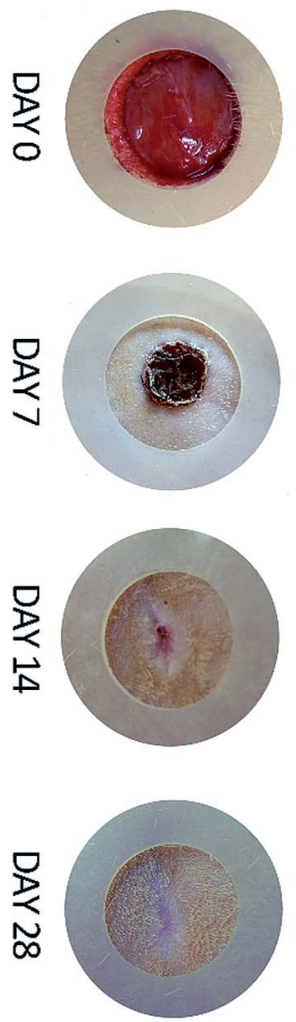
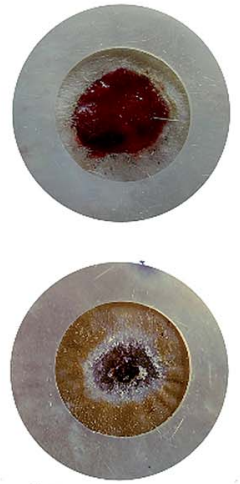

DM blank
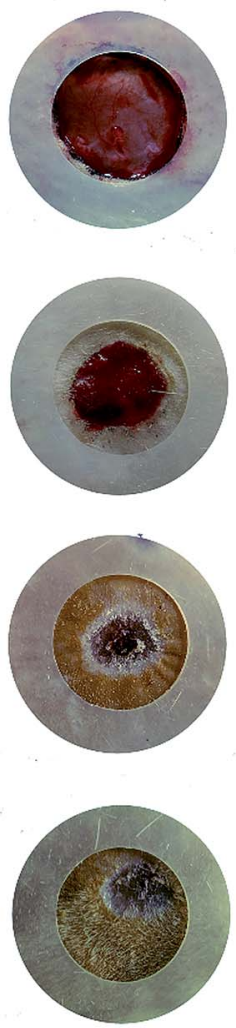

B

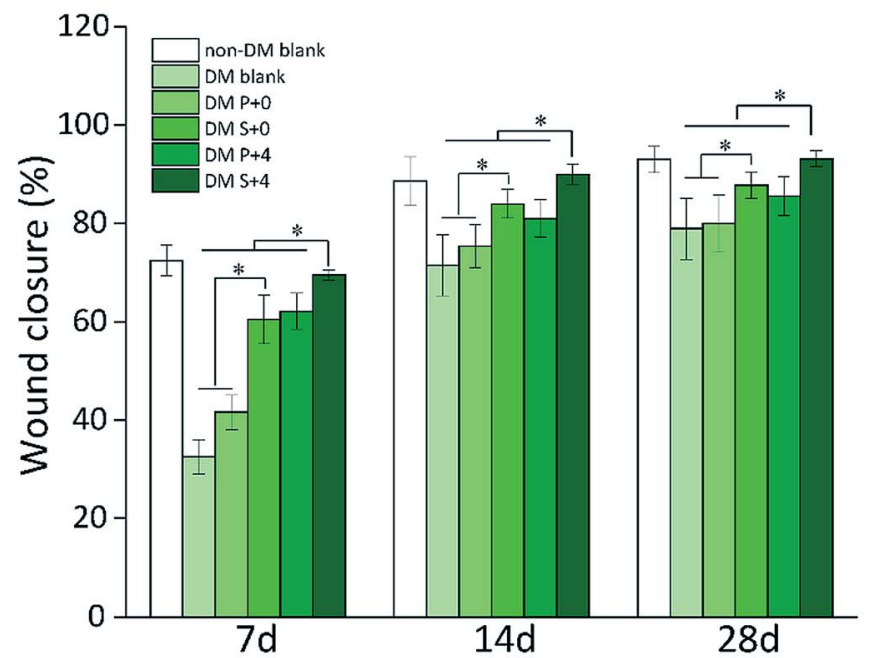

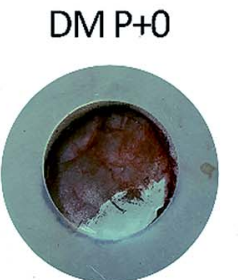

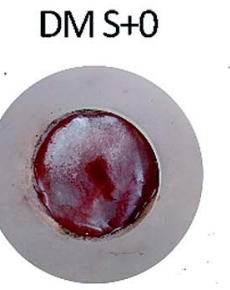

DM P+4

DMS+4
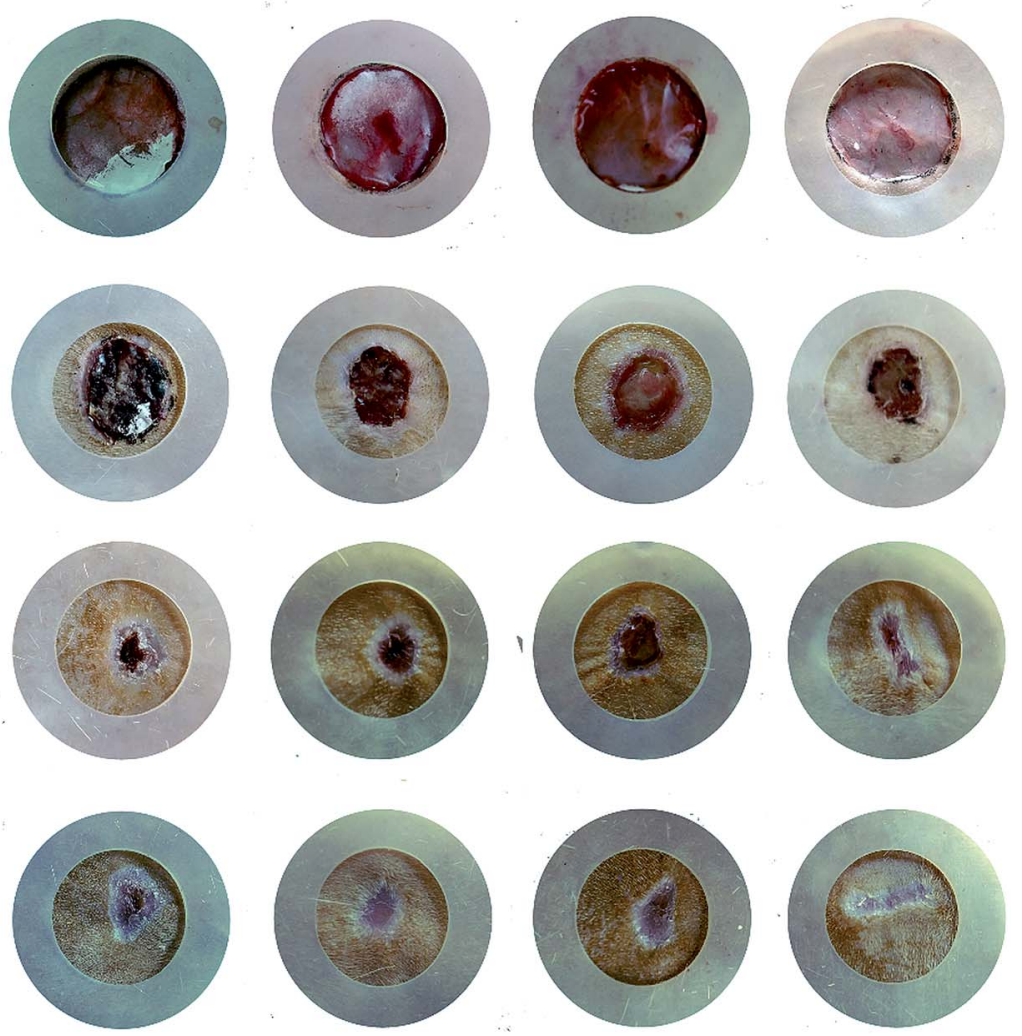

Fig. 5 (A) The full-thickness cutaneous wound model; wounds with a diameter of $18 \mathrm{~mm}$ were created on the back of each rat. (B) Timedependent changes in the wound closure determined using image analysis software, ${ }^{*} p<0.05$. (C) Representative images of the wounds were tracked at day 7, day 14, and day 28 .

\subsection{Oxidative stress at the wound site}

In order to further confirm the protective role of the S-PLGA scaffold from the oxidative stress generated at the wound site, the levels of the ROS were determined at day 7 (Fig. 6). The levels of the ROS were significantly higher in the DM blank group and the PLGA scaffold treated (DM P+0) group when compared to the S-PLGA scaffold treated (DM S +0 ) group. Additionally, decreased ROS levels were found in the HB-EGF treated (DM $\mathrm{P}+4$, DM S+4) group wounds when compared to the non-HBEGF treated (DM blank, DM P+0, DM S+0) group wounds. 

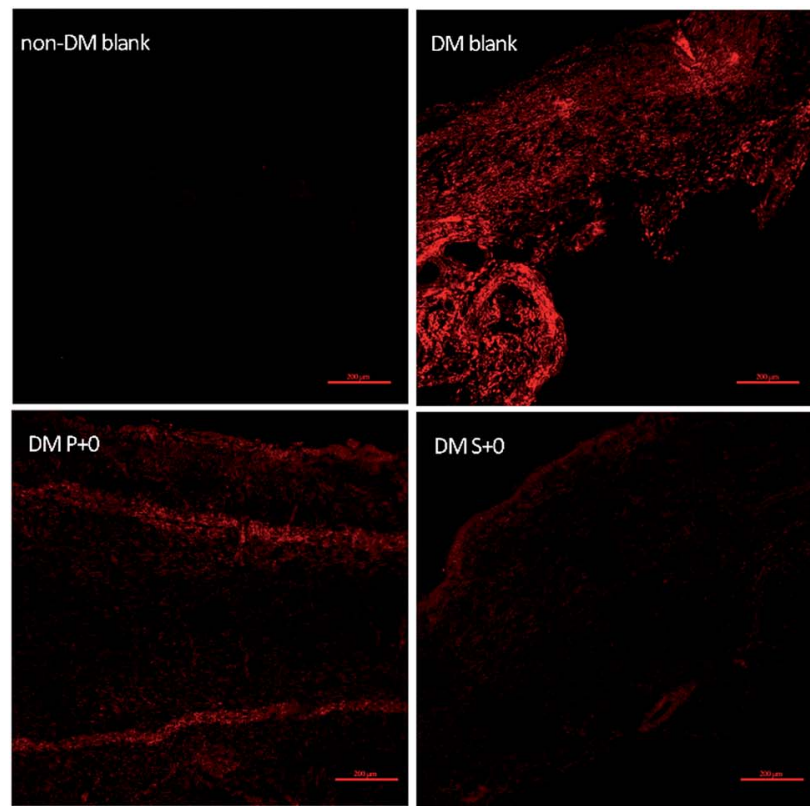

DMS+0
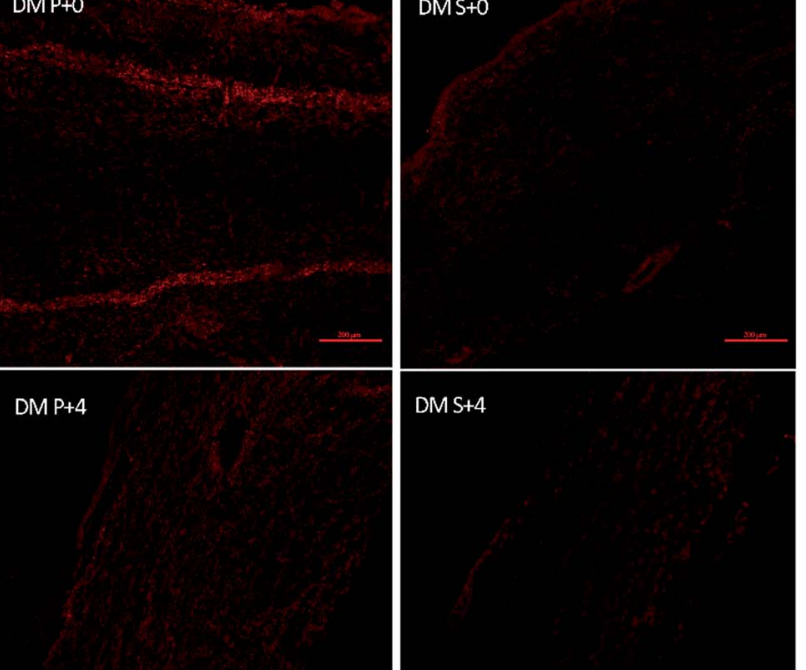

Fig. 6 Representative images of DHE staining of the sections to evaluate the ROS levels at the wound sites of various groups at day 7 post-surgery, scale bar $=200 \mu \mathrm{m}$.

\subsection{Cell viability in vivo}

The effect of HB-EGF on the proliferation of keratinocytes was assessed after 7 days post-wounding using Ki-67 (a marker of cell proliferation) immunofluorescence (Fig. 7). Meanwhile, the migration of keratinocytes was determined using stained $\beta 4$
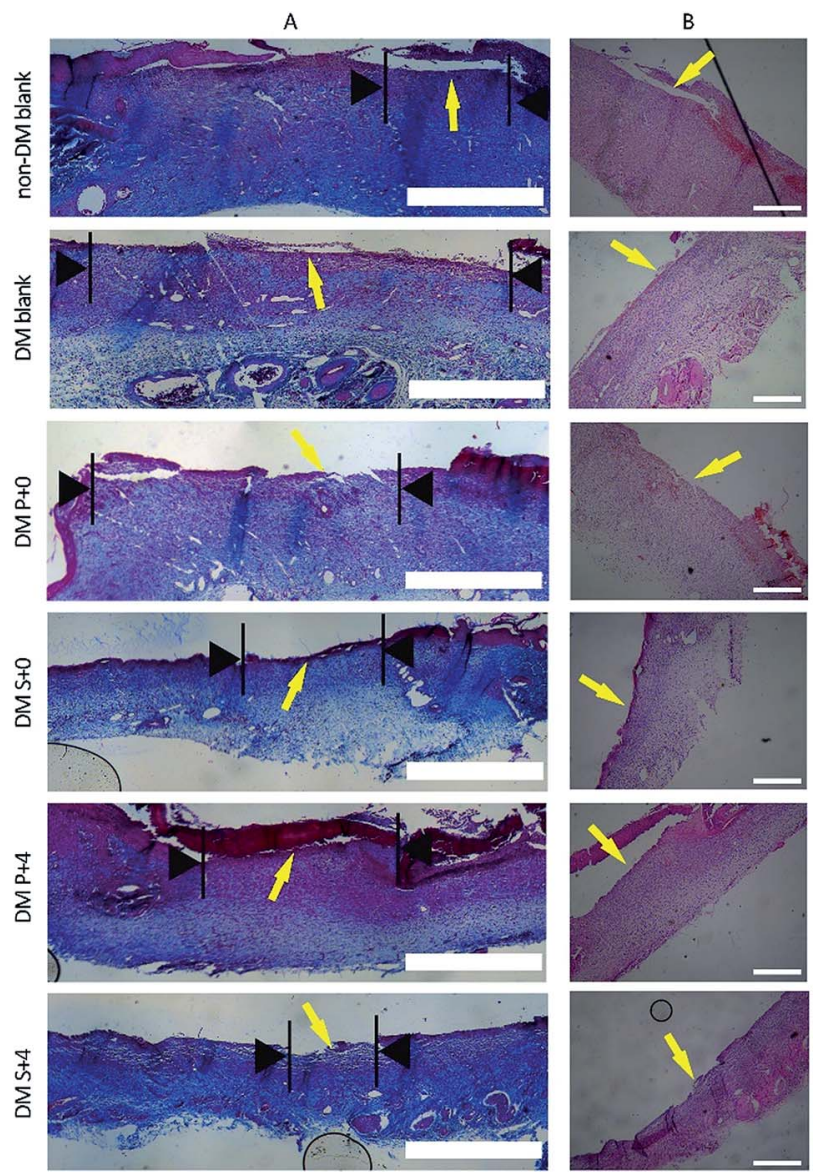

Fig. 8 Histological analysis of the wound regions for observing reepithelialization, 7 days after injury. (A) Staining with MTS. The black arrows indicate the wound surface that did not cover the new epithelia. Scale bars $=1 \mathrm{~mm}$. (B) Staining with HE. Scale bars $=500 \mu \mathrm{m}$.

integrin, which acts to activate Rac1 for coordinated cell motility during wound healing. The DM blank group and the $\mathrm{DM} \mathrm{P}+0$ group exhibited a double-negative throughout the wound region. The DM S +0 group induced a small amount of
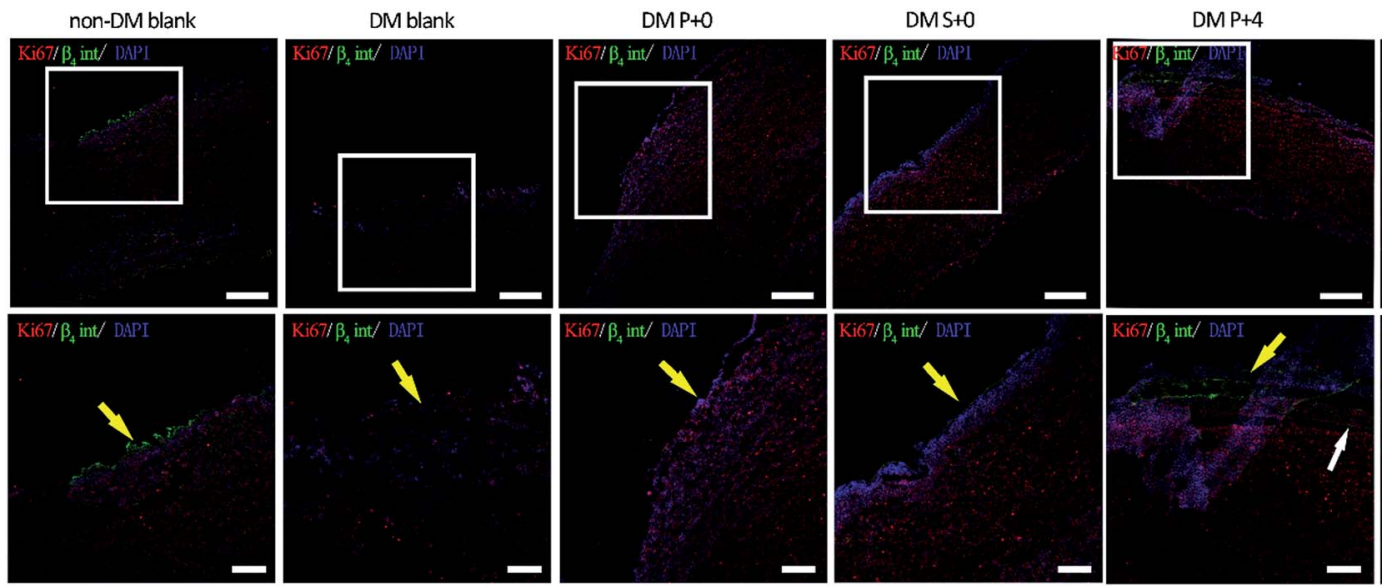

$\mathrm{DMS}+4$

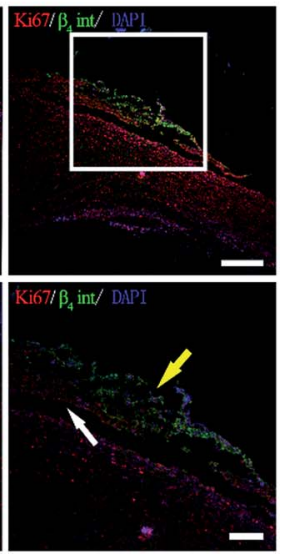

Fig. 7 Immunofluorescent staining of day 7 wound sections for $\beta 4$ integrin (green) and Ki-67 (red) with DAPI (blue) nuclear staining (yellow arrow: keratinocyte cells migrating, white arrow: keratinocyte cells proliferating). 
migration of nonproliferating (Ki-67-negative) cells and several related proliferating cells. The DM $\mathrm{P}+4$ group exhibited much more proliferation and cell migration ( $\beta 4$ integrin-positive) when compared with those in the other groups. Notably, the wounds in the DM S+4 group (HB-EGF + S-PLGA) showed a lot of migration of the proliferating cells (double-positive) when compared with those in the other groups, which suggested that 26SCS-modified PLGA scaffolds induced the migration of keratinocytes and related cell proliferation.

\subsection{Histopathological evaluation}

Histological analysis of the wound tissues, using H\&E staining and Masson's trichrome staining, 7 days after the injury, are presented in Fig. 8. As can be seen, the new epithelia did not cover the entire wound surface in all of the groups. We observed that the DM S +4 group had significantly decreased the uncovered area of the wound surface when compared with the other DM groups. By day 14, re-epithelialization was completed in all groups apart from the DM blank group (Fig. 9). In the DM P+0 group and the DM $\mathrm{P}+4$ group, the re-epithelialization was irregular, and there was a gap between the subcutaneous tissue and the dermis, which shows that the subcutaneous tissue was not completely healed. The other three groups showed complete and mature epidermis thickness, similar to non-DM blank group. Moreover, to further assess the effect of the HB-EGFloaded S-PLGA scaffold on wound healing, the contraction gap, epidermal thickness and collagen deposition were analyzed using Masson's trichrome staining (MTS), 28 days after the injury (Fig. 10). As shown in Fig. 10B, the contraction gap of the DM $\mathrm{S}+4$ group $(6.02 \pm 0.22 \mathrm{~mm})$ was significantly smaller than that in the $\mathrm{DM} \mathrm{P}+4$ group $(10.84 \pm 0.86 \mathrm{~mm})$.
There was no significant difference between the DM S+4 group and the non-DM blank group $(5.68 \pm 0.22 \mathrm{~mm})$. Interestingly, the contraction gap of the DM S+0 group $(12.65 \pm 1.29 \mathrm{~mm})$ was also significantly smaller than that of both the DM blank group $(17.25 \pm 0.61 \mathrm{~mm})$ and the DM P+0 group (16.39 $\pm 0.49 \mathrm{~mm})$. The wounds of the DM S+4 group demonstrated an increased thickness in the newly regenerated epidermis when compared with the other DM groups (see Fig. 10C). The epidermal layer thickness in the DM S+4 group was $98.18 \pm 21.84 \mu \mathrm{m}$, while the data for the DM P+4 group and the non-DM blank group were $75.34 \pm 13.94 \mu \mathrm{m}$ and $70.91 \pm 12.46 \mu \mathrm{m}$, respectively. It is noteworthy that the DM S+0 group $(89.55 \pm 15.71 \mu \mathrm{m})$ had an increased thickness in the newly regenerated epidermis when compared with the $\mathrm{DM} \mathrm{P}+4$ group. There was no significant difference between the DM blank group $(45.53 \pm 12.09 \mu \mathrm{m})$ and the DM P +0 group $(48.45 \pm 10.12 \mu \mathrm{m})$. The blank group, DM P+0 group, DM $\mathrm{S}+0$ group, DM $\mathrm{P}+4$ group and $\mathrm{DM} \mathrm{S}+4$ group had thicknesses of $22.7 \pm 2.78 \%, 27.8 \pm 8.05 \%, 45.9 \pm 7.89 \%, 49.9$ $\pm 7.07 \%$ and $58.3 \pm 2.71 \%, 28$ days post-surgery, respectively, and there was a significant difference between the DM S+0 group and the DM blank group and DM P+0 group. Furthermore, the effect of collagen deposition was analyzed (Fig. 10D). It was found that the collagen deposition percentage for the DM blank group, $\mathrm{DM} \mathrm{P}+0$ group, $\mathrm{DM} \mathrm{S}+0$ group, $\mathrm{DM} \mathrm{P}+4$ group and DM S+4 group were $22.7 \pm 2.78 \%, 27.8 \pm 8.05 \%, 45.9 \pm 7.89 \%$, $49.9 \pm 7.07 \%$ and $58.3 \pm 2.71 \%, 28$ days post-surgery, respectively, indicating that collagen deposition in the DM S+4 group was significantly higher than that in the other groups. There is a significant difference between the DM S+0 group and the DM blank group and DM $\mathrm{P}+0$ group.
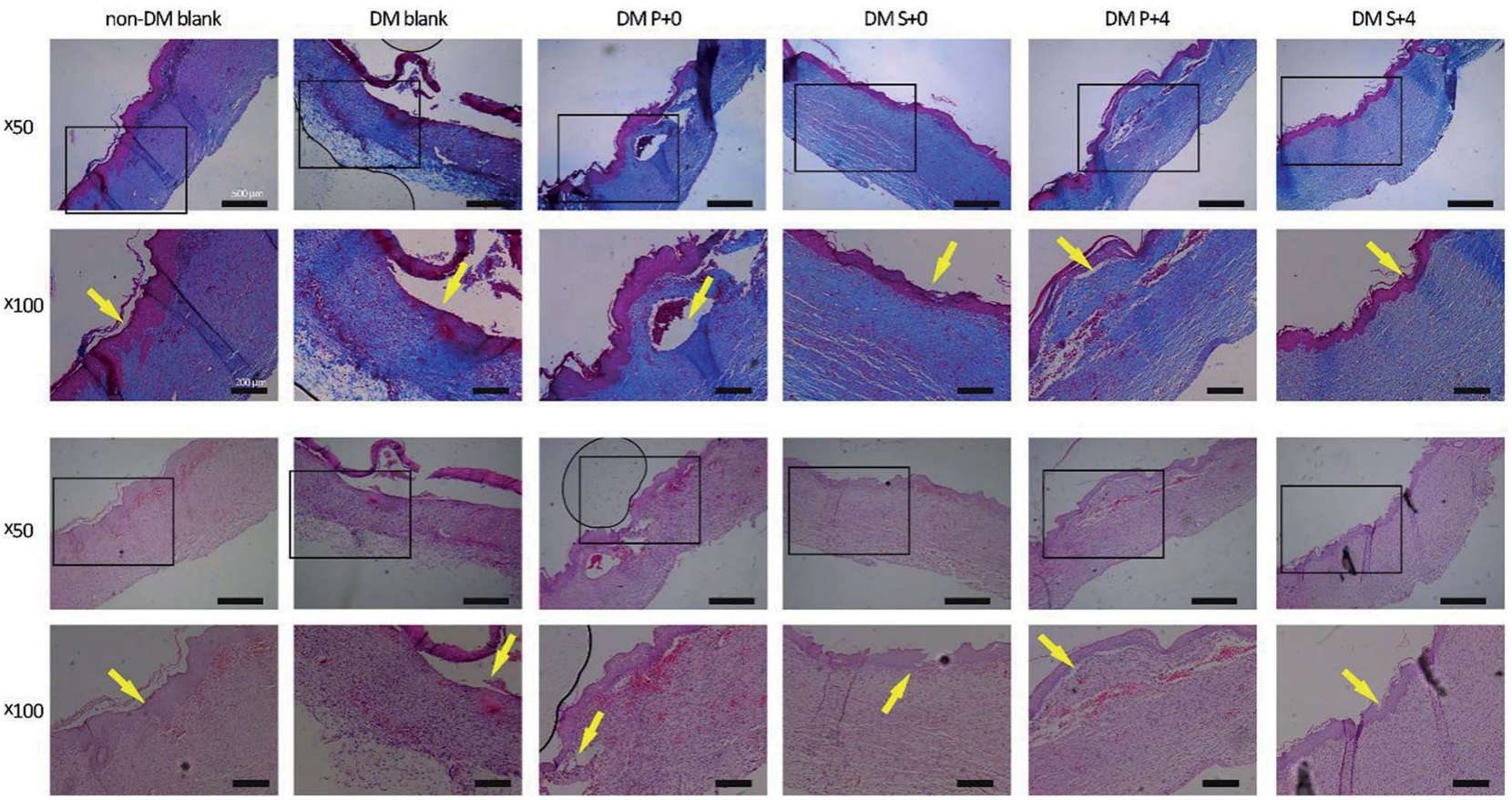

Fig. 9 Histological analysis of the wound regions for observing re-epithelialization, 14 days after injury. Staining with Masson's trichrome staining (MTS) and staining with hematoxylin and eosin (HE). 

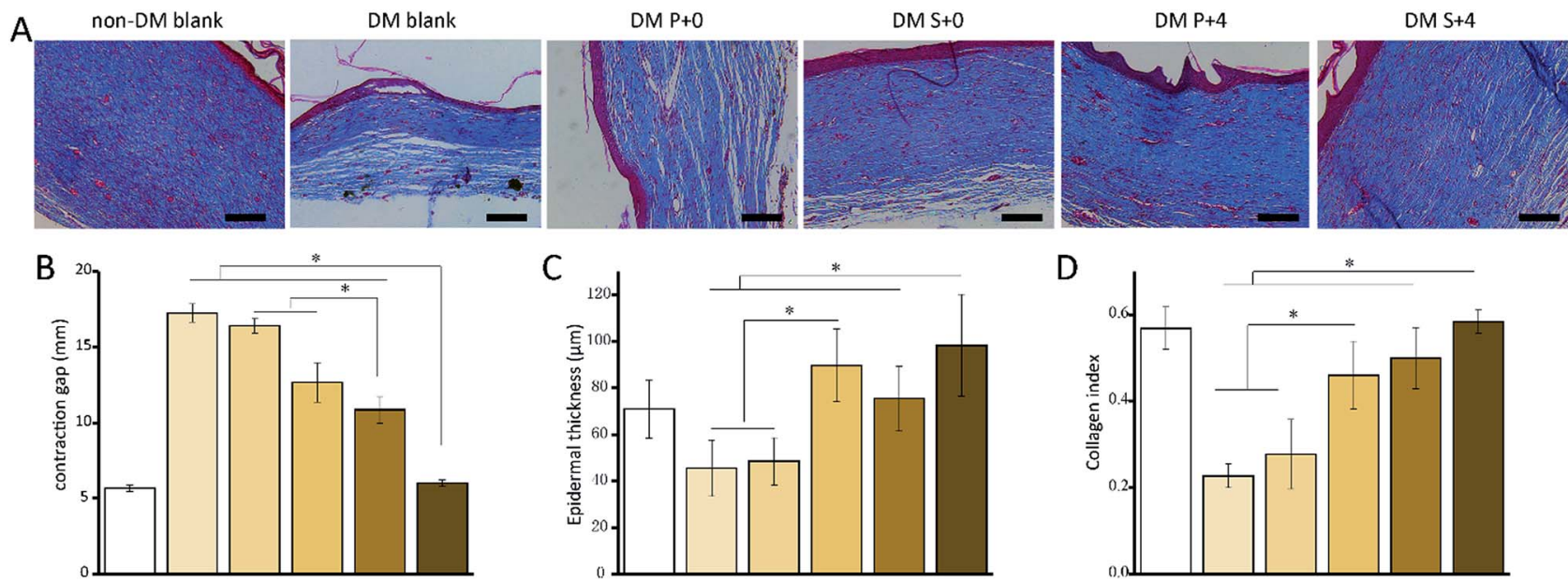

C

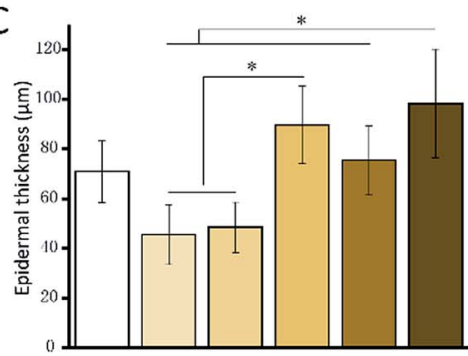

$\mathrm{D}$

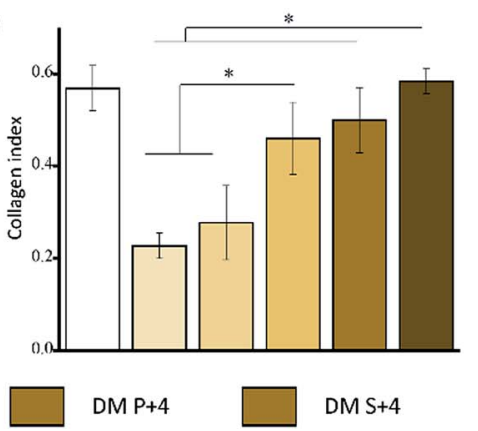

Fig. 10 Histological analysis of the wound regions 28 days after injury. (A) MTS for observing wound contraction. Scale bars: $100 \mu$ m. (B) The wound contraction was quantified by measuring the gap between the wound margins. (C) Quantification of the thickness of the epidermis. (D) Quantification of the collagen index. ${ }^{*} p<0.05$.

\section{Discussion}

Exogenous GFs applied in the body, especially in a wound environment with high proteolytic activity, have very short halflives. ${ }^{42}$ Consequently, numerous delivery systems that show protective and sustained release of the EGF family of proteins have been tested in animal models of diabetic wounds. ${ }^{43}$ In this study, we designed a 26SCS-modified scaffold (S-PLGA) after two steps of ammonification and sulfonation. As a result, PLGA and S-PLGA scaffold surfaces showed water contact angles of $128.99^{\circ}$ and $46.50^{\circ}$, respectively. One of the main focuses of PLGA being used for scaffolds is its hydrophobicity, because it does not allow biological fluids or cells to make contact with PLGA, which further obstructs cell infiltration. ${ }^{44} 2-\mathrm{N}, 6-\mathrm{O}-$ sulfated chitosan (26SCS), a hydrophilic polysaccharide, would drastically improve the hydrophilicity of the PLGA scaffold. Heparin and heparan sulfate, which have a high affinity to bind many growth factors, could protect them from proteolytic degradation and increase their bioactivity, which extends their half-lives. ${ }^{45,46}$ Moreover, heparin could increase the ability of some growth factors to bind to their cell receptor. ${ }^{47,48}$ 26SCS has a high affinity for heparin-binding growth factors and this can help to achieve controlled release. In our previous work, we demonstrated that heparin-like 26SCS had significant effects on BMP-2, VEGF and HB-EGF, even with a low dose of 26SCS. ${ }^{30-32}$ In this study, the S-PLGA scaffold was effectively bound to HB-EGF and decreased the rate of release of HB-EGF, especially the burst release, which was reduced from $44.3 \%$ to $31.7 \%$ (compared to the PLGA scaffold used as a control). Here, we demonstrate similar results to previous reports. Cao et al. developed a 26SCSmodified PCL scaffold for bone morphogenetic protein-2 (BMP2) delivery and the rate of release observed within $24 \mathrm{~h}$ was $30-$ $45 \% .{ }^{49}$ Moreover, at day 10 , a controlled and sustained release of HB-EGF of $73.8 \%$ was observed from the S-PLGA scaffold compared with $89.3 \%$ for the PLGA scaffold. Peng et al. fabricated a 26SCS-embedded PLGA nanofibrous matrix (SPLGA) as a delivery vehicle for HB-EGF. After 10 days, approximately $56 \%$ of the HB-EGF was released from the S-PLGA scaffold and the PLGA scaffold released a total of $83 \% .^{32}$ In contrast, our S-PLGA scaffold released more HB-EGF after 10 days, indicating the higher utilization efficiency of HB-EGF on the S-PLGA scaffold (Fig. 2E).

There has been growing recognition that many polysaccharides have scavenging activities for free radicals and can protect cells against oxidative damage induced by hydrogen peroxide. Moreover, it has been reported that the effect of super oxide dismutase (SOD), one of the representative antioxidant enzymes for scavenging radicals and reducing oxidative damage, could be improved by polysaccharides. 26SCS, a derivative of chitosan (CS), exhibits a great anti-antioxidant effect. Ronge Xing et al. studied the antioxidant capacity of different sulfated chitosan systems (236S, 6S, 3S, 36S). Low molecular weight chitosan sulfate showed effective scavenging activity towards superoxide radicals and hydroxyl radicals. ${ }^{25}$ However, up to now, few studies have focused on the antioxidant effect of chitosan for tissue regeneration. In this study, we assessed the antioxidant activity of 26SCS. It has a strong scavenging activity towards superoxide radicals, a moderate scavenging activity towards hydroxyl radicals and a low scavenging activity towards hydrogen peroxide (Fig. 3). We also demonstrated that the soluble 26SCS exhibited stronger protective effects in Ha-cat cells against $\mathrm{H}_{2} \mathrm{O}_{2}$-induced oxidative damage (Fig. 4A). From the results of the control group, it could be confirmed that 500 $\mu \mathrm{M} \mathrm{H}_{2} \mathrm{O}_{2}$ and $2 \mathrm{~h}$ incubation was sufficient to inhibit the proliferation of Ha-cat cells, and this could be caused by the ROS from $\mathrm{H}_{2} \mathrm{O}_{2}$. Liu et al. reported that the viability of Ha-cat cells decreased in a concentration-dependent and timedependent manner after exposure to $\mathrm{H}_{2} \mathrm{O}_{2}$. There was significant difference $(P<0.05)$ apart from at $50 \mu \mathrm{M} .{ }^{50}$ In this study, the results from the 26SCS groups showed significant protection for 


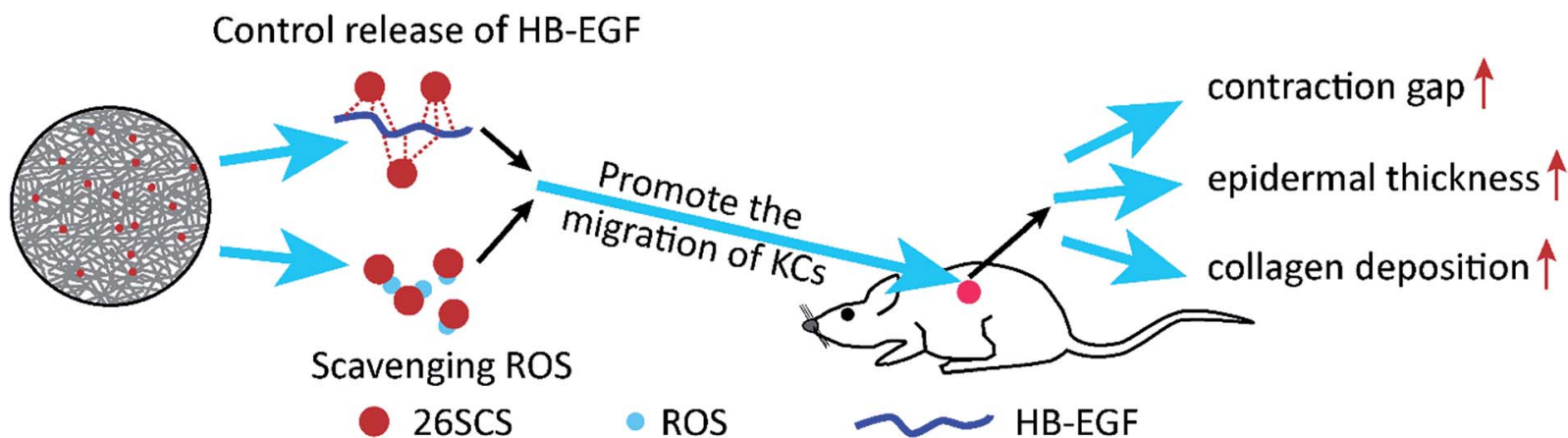

Fig. 11 A schematic diagram of the effect of the HB-EGF-loaded S-PLGA scaffold on wound healing. (1) The sustained and controlled release of HB-EGF from the S-PLGA scaffolds. (2) The scavenging activity of 26SCS towards ROS. (3) The synergistic effect of 26SCS and HB-EGF, promoting migration of the Ha-cat cells. (4) The HB-EGF-loaded S-PLGA scaffold enhanced the healing of full thickness excisional wounds in diabetic rats by affecting wound contraction, epithelial regeneration, and collagen deposition.

the viability of $\mathrm{H}_{2} \mathrm{O}_{2}$-induced Ha-cat cells, and this may benefit from the treatment with 26SCS, as well as their anti-oxidation. The cells cultured in the presence of the S-PLGA scaffold also showed significant protection against oxidative stress during proliferation (Fig. 4B).

To further confirm the effect of the HB-EGF-loaded S-PLGA scaffold on the behavior of wound healing in vivo, full thickness excisional wounds in diabetic rats were selected for this study. Sashwati et al. showed that certain concentrations (100$250 \mu \mathrm{M})$ of $\mathrm{H}_{2} \mathrm{O}_{2}$ are present at the normal wound site. Higher doses of $\mathrm{H}_{2} \mathrm{O}_{2}$ adversely influenced healing. ${ }^{51}$ Diabetic wounds are characterized by high levels of ROS, particularly $\mathrm{O}^{2-}$ and $\mathrm{H}_{2} \mathrm{O}_{2}$ which could lead to oxidative stress. ${ }^{52}$ In these circumstances, keratinocyte migration, homing and proliferation are impaired. ${ }^{53}$ It is worth noting that the ROS levels of the DM S+0 group had reduced to a degree (Fig. 5), and the DM S+0 group played a favorable role in proliferation (Fig. 6). These results suggest that 26SCS presumably protected the keratinocytes around the wound from ROS damage, and stimulated the keratinocytes to express the appropriate integrin receptors. As for the DM S+4 group, dihydroethidium (DHE) staining suggested that the ROS levels were effectively decreased when compared to the DM P+4 group at day 7. Moreover, the keratinocytes that stained double positive for $\beta 4$ integrin and Ki-67 protein were observed well beyond the wound margin in the DM S+4 group. As shown in Fig. 5, the diabetic wound healed faster, especially in the initial phase when 26SCS and HB-EGF were combined for treatment. Substantial dysregulation in the availability and activity of growth factors may cause keratinocyte functional impairment. ${ }^{54}$ The method of applying EGF to the wound site could stimulate keratinocyte proliferation with the mRNA expression levels of epiregulin, keratin, and loricrin significantly increasing. ${ }^{5,56}$ Locally prolonged bioavailability is a requirement for a significant EGF-mediated impact on wound closure. ${ }^{57}$ Experimental studies also suggest that EGF is able to enhance cell survival by controlling oxidative stress. ${ }^{58-60}$ What's more, the healing effect of EGF on diabetic wounds seems to be related to a noticeable improvement in the redox balance. ${ }^{61}$

The data for the histopathological evaluation showed that the HB EGF-loaded S-PLGA scaffold enhanced healing of the full thickness excisional wounds in diabetic rats by affecting wound contraction, epithelial regeneration, and collagen deposition (Fig. 11). The quantity and quality of collagen deposition is critical to wound contraction and revascularization. HB-EGF stimulates fibroblast proliferation and plays a critical role in the process of collagen remodeling..$^{62}$ Notably, there are also positive effects on wound contraction, epithelial regeneration, and collagen deposition when only the S-PLGA scaffold is used. Excessive ROS production leads to oxidative stress, which can cause impaired dermal fibroblast function. ${ }^{\mathbf{1 1}}$ Treatment with vitamin $\mathrm{C}$, an antioxidant, can reduce ROS, increase collagen deposition, as well as reduce apoptosis. ${ }^{19}$ Similar effects were observed with oral administration of an antioxidant; treatment with the mitochondria-targeted antioxidant SkQ1 reduced the ROS levels at the wound site and improved granulation tissue deposition. $^{21}$

\section{Conclusions}

In this study, we designed a 26SCS-modified scaffold (S-PLGA) after two steps of ammonification and sulfonation, which helped to improve the hydrophilicity. When the S-PLGA scaffold was developed for HB-EGF loading, controlled release of HBEGF was achieved. Furthermore, we assessed the antioxidant activity of 26SCS. It has a strong scavenging activity towards superoxide radicals, a moderate scavenging activity towards hydroxyl radicals and a low scavenging activity towards hydrogen peroxide. We also demonstrated that the soluble 26SCS exhibited stronger protective effects in Ha-cat cells against $\mathrm{H}_{2} \mathrm{O}_{2}$-induced oxidative damage. The S-PLGA scaffold played a role in protecting keratinocyte cells from oxidative damage, which led to acceleration of re-epithelialization. The wounds were almost completely healed 28 days after the application of the HB-EGF-loaded S-PLGA scaffold. Besides, the HB EGF-loaded S-PLGA scaffold enhanced healing of the full thickness excisional wounds in diabetic rats by affecting wound contraction, epithelial regeneration, and collagen deposition. Notably, there were also positive effects on wound contraction, epithelial regeneration, and collagen deposition when only the S-PLGA scaffold was used. By this token, these results indicate 
that the HB-EGF-loaded S-PLGA scaffold can promote the comprehensive and fast curing of cutaneous wounds.

\section{Conflicts of interest}

There are no conflicts of interests to declare.

\section{Acknowledgements}

The authors wish to express their gratitude for financial support from the National Natural Science Foundation of China (No. 31330028, No. 31470923), the National Natural Science Foundation of China for Innovative Research Groups (No. 51621002), the International Cooperation Project of Shanghai Science and Technique Committee (15520711100) and the Key Project of Logistic Scientific Research of PLA (BWS13C014). This study was also supported by the 111 Project (B14018).

\section{Notes and references}

1 J. E. Shaw, R. A. Sicree and P. Z. Zimmet, Diabetes Res. Clin. Pract., 2010, 87, 4-14.

2 J. B. Acosta, D. G. del Barco, D. C. Vera, W. Savigne, P. LopezSaura, G. G. Nieto and G. S. Schultz, Int. Wound J., 2008, 5, 530-539.

3 R. Blakytny and E. B. Jude, Int. J. Low. Extrem. Wounds, 2009, 8, 95-104.

4 Z. T. Bloomgarden, Diabetes Care, 2008, 31, 372-376.

$5 \mathrm{H}$. Brem and M. Tomic-Canic, J. Clin. Invest., 2007, 117, 1219-1222.

6 M. Muller, C. Trocme, B. Lardy, F. Morel, S. Halimi and P. Y. Benhamou, Diabet. Med., 2008, 25, 419-426.

7 E. Tsourdi, A. Barthel, H. Rietzsch, A. Reichel and S. R. Bornstein, BioMed Res. Int., 2013, 2013, 385641.

8 L. I. Moura, A. M. Dias, E. Carvalho and H. C. de Sousa, Acta Biomater., 2013, 9, 7093-7114.

9 B. Kunkemoeller and T. R. Kyriakides, Antioxidants Redox Signal., 2017, 27, 827-838.

10 N. Spravchikov, G. Sizyakov, M. Gartsbein, D. Accili, T. Tennenbaum and E. Wertheimer, Diabetes, 2001, 50, 1627-1635.

11 B. Y. Kim, M. J. Han and A. S. Chung, Free Radical Biol. Med., 2001, 30, 686-698.

12 C. C. E. Lan, C. S. Wu, H. Y. Kuo, S. M. Huang and G. S. Chen, Br. J. Dermatol., 2009, 160, 1206-1214.

13 W. Droge, Physiol. Rev., 2002, 87, 47-95.

14 T. Hellwig-Bürgel, D. P. Stiehl, A. E. Wagner, E. Metzen and W. Jelkmann, J. Interferon Cytokine Res., 2005, 25, 297-310.

15 S. Frank, H. Kampfer, C. Wetzler and J. Pfeilschifter, Kidney Int., 2002, 61, 882-888.

16 M. A. Loots, E. N. Lamme, J. Zeegelaar, J. R. Mekkes, J. D. Bos and E. Middelkoop, J. Invest. Dermatol., 1998, 111, 850-857.

17 A. L. Weinstein, F. D. Lalezarzadeh, M. A. Soares, P. B. Saadeh and D. J. Ceradini, Wound Repair Regen., 2015, 23, 14-21.

18 P. S. Rabbani, A. Zhou, Z. M. Borab, J. A. Frezzo, N. Srivastava, H. T. More, W. J. Rifkin, J. A. David,
S. J. Berens, R. Chen, S. Hameedi, M. H. Junejo, C. Kim, R. A. Sartor, C. F. Liu, P. B. Saadeh, J. K. Montclare and D. J. Ceradini, Biomaterials, 2017, 132, 1-15.

19 Y. H. Lee, J. J. Chang, C. T. Chien, M. C. Yang and H. F. Chien, Exp. Diabetes Res., 2012, 2012, 504693.

20 M. Ram, V. Singh, S. Kumawat, V. Kant, S. K. Tandan and

D. Kumar, Int. Immunopharm., 2016, 30, 137-149.

21 I. A. Demyanenko, V. V. Zakharova, O. P. Ilyinskaya, T. V. Vasilieva, A. V. Fedorov, V. N. Manskikh, R. A. Zinovkin, O. Y. Pletjushkina, B. V. Chernyak, V. P. Skulachev and E. N. Popova, Oxid. Med. Cell. Longevity, 2017, 6408278.

22 W. M. Xie, P. X. Xu and Q. Liu, Bioorg. Med. Chem. Lett., 2001, 11, 1699-1701.

23 F. Khodagholi, B. Eftekharzadeh, N. Maghsoudi and P. F. Rezaei, Mol. Cell. Biochem., 2010, 337, 39-51.

24 F. Karadeniz, M. Artan, C.-S. Kong and S.-K. Kim, Carbohydr. Polym., 2010, 82, 143-147.

25 R. Xing, S. Liu, Z. Guo, H. Yu, P. Wang, C. Li, Z. Li and P. Li, Bioorg. Med. Chem., 2005, 13, 1573-1577.

26 J. Wang, H. Guo, J. Zhang, X. Wang, B. Zhao, J. Yao and Y. Wang, Carbohydr. Polym., 2010, 81, 897-905.

27 Z. J. Wang, J. H. Xie, L. J. Kan, J. Q. Wang, M. Y. Shen, W. J. Li, S. P. Nie and M. Y. Xie, Int. J. Biol. Macromol., 2015, 80, 410417.

28 A. Kerschenmeyer, O. Arlov, V. Malheiro, M. Steinwachs, M. Rottmar, K. Maniura-Weber, G. Palazzolo and M. Zenobi-Wong, Biomater. Sci., 2017, 5, 1756-1765.

29 R. R. Chen and D. J. Mooney, Pharm. Res., 2003, 20, 11031112.

30 L. Cao, J. Wang, J. Hou, W. Xing and C. Liu, Biomaterials, 2014, 35, 684-698.

31 Y. Yu, J. Chen, R. Chen, L. Cao, W. Tang, D. Lin, J. Wang and C. Liu, ACS Appl. Mater. Interfaces, 2015, 7, 9982-9990.

32 X. Peng, Y. Yu, Z. Wang, X. Zhang, J. Wang and C. Liu, RSC Adv., 2017, 7, 43161-43171.

33 Y. Pan, J. Chen, Y. Yu, K. Dai, J. Wang and C. Liu, Biomater. Sci., 2018, 6, 431-439.

34 J. Tavakoli, J. Mech. Behav. Biomed. Mater., 2017, 65, 373-382. 35 M. Nishikimi, N. Appaji Rao and K. Yagi, Biochem. Biophys. Res. Commun., 1972, 46, 849-854.

36 P. Seedevi, M. Moovendhan, S. Vairamani and A. Shanmugam, Int. J. Biol. Macromol., 2017, 99, 519-529.

37 B. Hsu, I. M. Coupar and K. Ng, Food Chem., 2006, 98, 317328.

38 C. Liu, C. Wang, Z. Xu and Y. Wang, Process Biochem., 2007, 42, 961-970.

39 W. Wang, S. Lin, Y. Xiao, Y. Huang, Y. Tan, L. Cai and X. Li, Life Sci., 2008, 82, 190-204.

40 F. Silver, Y. Kato, M. Ohno and A. Wasserman, J. Long Term Eff. Med. Implants, 1992, 2, 165-198.

41 M. Sun, J. Deng and C. Gao, J. Colloid Interface Sci., 2015, 448, 231-237.

42 B. Mast and G. Schultz, Wound Repair Regen., 1996, 4, 411420.

43 T. N. Demidova-Rice, M. R. Hamblin and I. M. Herman, Adv. Skin Wound Care, 2012, 25, 349-370. 
44 F. A. Sheikh, H. W. Ju, B. M. Moon, O. J. Lee, J.-H. Kim, H. J. Park, D. W. Kim, D.-K. Kim, J. E. Jang, G. Khang and C. H. Park, J. Regen. Med. Tissue Eng., 2016, 10, 209-221.

45 G. S. Schultz and A. Wysocki, Wound Repair Regen., 2009, 17, 153-162.

46 R. Hynes, Science, 2009, 326, 1216-1219.

47 D. H. Damon, R. R. Lobb, P. A. D'Amore and J. A. Wagner, J. Cell. Physiol., 1989, 138, 221-226.

48 T. Spivak-Kroizman, M. A. Lemmon, I. Dikic, J. E. Ladbury, D. Pinchasi, J. Huang, M. Jaye, G. Crumley, J. Schlessinger and I. Lax, Cell, 1994, 79, 1015-1024.

49 L. Cao, Y. Yu, J. Wang, J. A. Werkmeister, K. M. McLean and C. Liu, Mater. Sci. Eng., C, 2017, 74, 298-306.

50 L. Liu, H. Xie, X. Chen, W. Shi, X. Xiao, D. Lei and J. Li, Clin. Exp. Dermatol., 2012, 37, 772-780.

51 S. Roy, S. Khanna, K. Nallu, T. K. Hunt and C. K. Sen, Mol. Ther., 2006, 11, 211-220.

52 S. Dhall, D. C. Do, M. Garcia, J. Kim, S. H. Mirebrahim, J. Lyubovitsky, S. Lonardi, E. A. Nothnagel, N. Schiller and M. Martins-Green, J. Diabetes Res., 2014, 2014, 562625.

53 V. Falanga, Lancet, 2005, 366, 1736-1743.

54 S. E. Thomson, S. V. McLennan and S. M. Twigg, Expet Rev. Clin. Immunol., 2006, 2, 403-418.
55 K. Hashimoto, S. Higashiyama, H. Asada, E. Hashimura, T. Kobayashi, K. Sudo, T. Nakagawa, D. Damm, K. Yoshikawa and N. Taniguchi, J. Biol. Chem., 1994, 269, 20060-20066.

56 K. P. Xu, Y. F. Li, A. V. Ljubimov and F. S. X. Yu, Diabetes, 2009, 58, 1077-1085.

57 A. Buckley, J. M. Davidson, C. D. Kamerath, T. B. Wolt and S. C. Woodward, Proc. Natl. Acad. Sci. U. S. A., 1985, 82, 7340-7344.

58 J. Berlanga, P. Prats, D. Remirez, R. Gonzalez, P. LopezSaura, J. Aguiar, M. Ojeda, J. J. Boyle, A. J. Fitzgerald and R. J. Playford, Am. J. Pathol., 2002, 161, 373-379.

59 M. E. Caballero, J. Berlanga, D. Ramirez, P. Lopez-Suara, R. Gozalez, D. N. Floyd, T. Marchbank and R. J. Playford, Gut, 2001, 48, 34-40.

60 Z. Hu, C. Chen, J. Yang, Z. Zhou, Z. Song and Z. Wang, Int. J. Ophthalmol., 2014, 7, 413-420.

61 A. G. Ojalvo, J. B. Acosta, Y. M. Mari, M. F. Mayola, C. V. Perez, W. S. Gutierrez, Marichal, II, E. A. Seijas, A. M. Kautzman, A. E. Pacheco and D. G. Armstrong, Int. Wound J., 2017, 14, 214-225.

62 R. Kajanne, P. Miettinen, A. Mehlem, S. K. Leivonen, M. Birrer, M. Foschi, V. M. Kahari and S. Leppa, J. Cell. Physiol., 2007, 212, 489-497. 